\title{
Linear Redundancy of Information Carried by the Discrete Wigner Distribution
}

\author{
Cédric Richard, Member, IEEE
}

\begin{abstract}
The discrete Wigner distribution (WD) encodes information in a redundant fashion since it derives $N$ by $N$ representations from $N$-sample signals. The increased amount of data often prohibits its effective use in applications such as signal detection, parameter estimation, and pattern recognition. As a consequence, it is of great interest to study the redundancy of information it carries. Recently, Richard and Lengellé have shown that linear relations connect the time-frequency samples of the discrete WD. However, up until now, such a redundancy has still not been algebraically characterized. In this paper, the problem of the redundancy of information carried by the discrete cross WD of complex-valued signals is addressed. We show that every discrete WD can be fully recovered from a small number of its samples via a linear map. The analytical expression of this linear map is derived. Special cases of the auto WD of complex-valued signals and realvalued signals are considered. The results are illustrated by means of computer simulations, and some extensions are pointed out.
\end{abstract}

Index Terms-Algebraic characterization, discrete Wigner distribution, linear redundancy, time-frequency analysis.

\section{INTRODUCTION}

$\mathbf{T}$ HE WIGNER distribution (WD) plays an important role in signal analysis and signal processing since it extends the usual spectral analysis by making it time dependent. Its usefulness partly derives from the fact that it satisfies many desirable mathematical properties [3], [6], [9], [18]. For a practical implementation on a digital computer, the WD must be reformulated in a discrete-time, discrete-frequency setting. There are several different approaches to extending its definition to the discrete case. For example, a group theoretic definition has been used in [28]. The resulting discrete distribution satisfies several mathematical properties analogous to those satisfied by the continuous distribution. However, its definition depends on the parity of the signal length. In [20], an axiomatic approach has been developed. Under an alternative definition for the WD, it is shown that the discrete distribution exists only for odd-length signals and corresponds to the one originally defined in [28]. In addition, this approach has been extended in [21] to derive a discrete Cohen class. There has also been a lot more work investigating discretization methods [22] and techniques for overcoming the inherent aliasing effects [2], [4], [19]. Among the various defi-

Manuscript received March 28, 2000; revised July 23, 2001. The associate editor coordinating the review of this paper and approving it for publication was Prof. Colin F. Cowan.

The author is with the Laboratoire de Modélisation et Sûreté des Systèmes, Université de Technologie de Troyes, Troyes, France (e-mail: cedric.richard@utt.fr).

Publisher Item Identifier S 1053-587X(01)09234-0. nitions for the discrete WD, the most commonly used form is

$$
\begin{aligned}
W_{x y}(n, m) \triangleq \sum_{k} R_{x y}(n, k) \exp \left(-\frac{2 j \pi m k}{N}\right) \\
\quad \text { with }(n, m) \in\{0, \ldots, N-1\}^{2} .
\end{aligned}
$$

Here, $x$ and $y$ are $N$-sample complex-valued signals, and the local cross correlation $R_{x y}$ is given by $R_{x y}(n, k) \triangleq x(n+$ k) $y^{*}(n-k)$ if $(n+k, n-k) \in\{0, \ldots, N-1\}^{2}$ and 0 otherwise. $W_{x y}$ is the cross WD of two signals $x, y$. The corresponding auto WD is then defined as $W_{x} \triangleq W_{x x}$. This discrete distribution can be computed in a straightforward manner using the discrete Fourier transform. The resulting time-frequency (TF) representation is a halfband representation, meaning that frequency components of signals are computed in the normalized range of $[-(1 / 4), 1 / 4]$.

Since $N \times N$ representations are derived from $N$-sample signals, it is clear that the discrete WD defined by (1) encodes information in a redundant fashion. The increased amount of data often prohibits the effective use of this distribution in applications such as signal detection, parameter estimation, and pattern recognition. Various methods of data reduction have been proposed. For example, the singular value decomposition [8] of the $N \times N$ matrix of samples $W_{x}(n, m)$ has been used in [13]-[17], and techniques based on the statistical learning theory of Vapnik [29] have been proposed in [24], [25], and [27]. In [1] and [5], scatter criteria have been considered for the dimensionality reduction of a TF classifier. Even if it is of great interest to study the redundancy of information carried by the discrete WD, as far as we know, this problem has not been completely resolved. In [23], [26], and [27], Richard and Lengellé prove that linear relations connect the samples of the discrete auto WD. This means that sets of TF locations exist, and are denoted $\mathcal{L}$, so that every sample $W_{x}(n, m)$ with $(n, m) \in\{0, \ldots, N-1\}^{2}$ can be expressed as a linear combination of the elements of $\left\{W_{x}(n, m)\right.$ : $(n, m) \in \mathcal{L}\}$, which is denoted $\mathcal{G}_{\mathcal{L}}$, for all $x \in \mathbb{C}^{N}$. However, up to now, $\mathcal{L}$ and the associated linear map $\mathbf{T}_{\mathcal{L}}$ that allows the generation of every sample $W_{x}(n, m)$ of the discrete auto WD from $\mathcal{G}_{\mathcal{L}}$ have still not been characterized.

The general subject of this paper is the algebraic study of the linear redundancy of the information carried by the discrete WD defined by (1). We wish to formally characterize the elements and the cardinality of $\mathcal{L}$ and, given $\mathcal{L}$, to derive the associated linear map $\mathbf{T}_{\mathcal{L}}$. The paper is organized as follows. Section II provides a study of the linear redundancy of $W_{x y}$ for all $x$, $y \in \mathbb{C}^{N}$. Section III considers the special cases of $W_{x}$ where $x$ is any complex-valued signal or any real-valued signal. In Section IV, results are illustrated by means of computer simulations. 
Finally, some further areas of investigation are suggested in Section V.

\section{LiNEAR REDUNDANCY OF THE DisCRETE CROSS WD OF COMPLEX-VALUED Signals}

As a basis for subsequent development, Section II provides a brief review of linear spaces, and the concept of induced spaces is introduced [10]. Next, an algebraic characterization of the linear redundancy of information carried by $W_{x y}$ with $x, y \in$ $\mathbb{C}^{N}$ is proposed.

\section{A. Linear Signal Spaces, Induced Spaces, and Bases}

A linear signal space $\mathcal{S}$ over the field $\mathbb{C}$ is a collection of signals that satisfies the following linearity property: If $x \in \mathcal{S}$ and $y \in \mathcal{S}$, then $(\alpha x+\beta y) \in \mathcal{S}$ for arbitrary complex coefficients $\alpha, \beta$. Let $\left\{s_{q}\right\}$ be a nonempty set of elements of $\mathcal{S}$. We will say that it is a basis for $\mathcal{S}$ if it is linearly independent and if it generates $\mathcal{S}$. The basis $\left\{s_{q}\right\}$ is orthonormal if $\left\langle s_{q} \mid s_{q^{\prime}}\right\rangle=\delta_{q q^{\prime}}$, where $\delta_{q q^{\prime}}$ is the Kronecker delta symbol. Then, every $x \in \mathcal{S}$ can be represented as $x=\sum_{q} \alpha_{q} s_{q}$, where $\alpha_{q}=\left\langle x \mid s_{q}\right\rangle=\sum_{n=0}^{N-1} x(n) s_{q}^{*}(n)$ is the inner product of $x$ and $s_{q}$. In this section, we will consider that $\mathcal{S}$ is the linear space of $N$-sample complex-valued signals over the field $\mathbb{C}$.

In the process of discrete WD calculation as expressed by (1), two maps $\mathrm{r}$ and $\mathrm{w}$ can be distinguished:

$$
(x, y) \stackrel{\mathbf{r}}{\longmapsto} R_{x y} \stackrel{\mathbf{w}}{\longmapsto} W_{x y}
$$

such that $W_{x y} \triangleq($ wor $)(x, y)$. Here, $R_{x y}$ and $W_{x y}$ denote $N \times$ $N$ matrices whose components are $R_{x y}(n, k)$ and $W_{x y}(n, m)$, respectively. Let $\mathcal{R}$ and $\mathcal{W}$ be the images of $\mathbf{r}$ and $(\mathbf{w} \circ \mathbf{r})$, respectively. These spaces are then given by

$$
\begin{aligned}
& \mathcal{R} \triangleq\left\{R_{x y}: R_{x y}=\mathbf{r}(x, y) \text { with } x, y \in \mathbb{C}^{N}\right\} \\
& \mathcal{W} \triangleq\left\{W_{x y}: W_{x y}=(\mathbf{w} \circ \mathbf{r})(x, y) \text { with } x, y \in \mathbb{C}^{N}\right\} .
\end{aligned}
$$

We note that the space $\mathcal{R}$ is not a linear space: linear combinations of local correlations are not valid local correlations. We now associate with $\mathcal{R}$ the linear space $\tilde{\mathcal{R}}$ over the field $\mathbb{C}$ of all linear combinations of local correlations $R_{x y}$ with $x$, $y \in \mathbb{C}^{N}$. In [10], $\tilde{\mathcal{R}}$ is called "induced $R_{x y}$-domain space." For this space, the definition of the inner product is given by $\left\langle R_{x y} \mid R_{x^{\prime} y^{\prime}}\right\rangle=\sum_{n} \sum_{k} R_{x y}(n, k) R_{x^{\prime} y^{\prime}}^{*}(n, k)$. Let $\left\{\tilde{R}_{q}\right\}$ be a basis for $\tilde{\mathcal{R}}$. We will define the dimension of $\tilde{\mathcal{R}}$, which is denoted $\operatorname{dim}(\tilde{\mathcal{R}})$, as the cardinality of $\left\{\tilde{R}_{q}\right\}$ [11], [12]. Again, the space $\mathcal{W}$ is not a linear space. Let $\mathcal{W}$ be the induced $W_{x y}$-domain space over the field $\mathbb{C}$ of all linear combinations of $W_{x y}$ with $x, y \in \mathbb{C}^{N}$. Here, the inner product is expressed as $\left\langle W_{x y} \mid W_{x^{\prime} y^{\prime}}\right\rangle=\sum_{n} \sum_{m} W_{x y}(n, m) W_{x^{\prime} y^{\prime}}^{*}(n, m)$. We will define $\operatorname{dim}(\tilde{\mathcal{W}})$ as the cardinality of $\left\{\tilde{W}_{q}\right\}$, where $\left\{\tilde{W}_{q}\right\}$ is a basis for $\tilde{\mathcal{W}}$. In the following, we will use bases of the linear spaces $\tilde{\mathcal{R}}$ and $\tilde{\mathcal{W}}$ to characterize the elements of the underlying spaces $\mathcal{R}$ and $\mathcal{W}$. For example, we note that the number of linearly independent samples $W_{x y}(n, m)$ of every $W_{x y} \in \mathcal{W} \subset \tilde{\mathcal{W}}$ is upper bounded by the dimension of the space $\tilde{\mathcal{W}}$. This means that the maximum number of samples

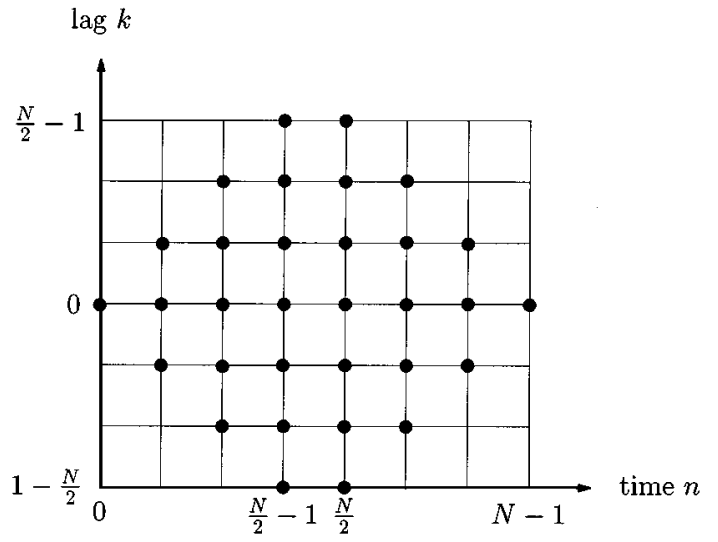

Fig. 1. Cross correlation domain support region defined as follows: $\mathcal{J}=$ $\left\{(n, k) \in \mathbb{Z}^{2}:(n+k, n-k) \in\{0, \ldots, N-1\}^{2}\right\}$.

$W_{x y}(n, m)$ that are sufficient to reconstruct every $W_{x y}$ via a linear map is equal to $\operatorname{dim}(\tilde{\mathcal{W}})$.

\section{B. Redundancy of Information Carried by $W_{x y}$}

Following this brief presentation of the induced spaces $\tilde{\mathcal{R}}$ and $\tilde{\mathcal{W}}$, we will now study the linear redundancy of information encoded by the discrete cross WD of complex-valued signals. To further establish that linear relations connect the samples $W_{x y}(n, m)$ of $W_{x y}$, we first have to determine a basis for $\tilde{\mathcal{W}}$. We will use it to calculate $\operatorname{dim}(\tilde{\mathcal{W}})$. By construction, the cross correlation $R_{x y}$ can be represented as

$$
R_{x y}=\sum_{(n, k) \in \mathcal{J}} x(n+k) y^{*}(n-k) R_{\Delta_{(n+k)} \Delta_{(n-k)}}
$$

where $\mathcal{J}$ is the set of pairs $(n, k)$ such that both $n+k \in\{0, \ldots, N-1\}$ and $n-k \in\{0, \ldots, N-1\}$, as depicted schematically in Fig. 1 , and $\Delta_{p}$ is the pulse defined by $\Delta_{p}(m)=1$ if $m=p$ and 0 otherwise, with $m$, $p \in\{0, \ldots, N-1\}$. Hence, $\left\{R_{\Delta_{(n+k)} \Delta_{(n-k)}}:(n, k) \in \mathcal{J}\right\}$ generates the space $\tilde{\mathcal{R}}$. Furthermore, simple calculus shows that $\left\langle R_{\Delta_{(n+k)} \Delta_{(n-k)}} \mid R_{\Delta_{\left(n^{\prime}+k^{\prime}\right)} \Delta_{\left(n^{\prime}-k^{\prime}\right)}}\right\rangle$ equals $\delta_{n n^{\prime}} \delta_{k k^{\prime}}$. From this, we conclude at once that our family is an orthonormal basis for $\tilde{\mathcal{R}}$. As defined by (1), the discrete WD at time-index $n$ is the discrete Fourier transform of the local correlation at time-index $n$. This implies that the linear map $\mathrm{w}$ is an isomorphism of $\tilde{\mathcal{R}}$ into $\tilde{\mathcal{W}}$ that preserves orthonormality. It follows that the family of generators $\left\{W_{\Delta_{(n+k)} \Delta_{(n-k)}}:(n, k) \in \mathcal{J}\right\}$ is an orthonormal basis for $\tilde{\mathcal{W}}$. In addition, we note that the cross WD of any complex-valued signals $x$ and $y$ can be expanded in the following form:

$$
W_{x y}=\sum_{(n, k) \in \mathcal{J}} x(n+k) y^{*}(n-k) W_{\Delta_{(n+k)} \Delta_{(n-k)}} .
$$

By definition, any element of the linear space $\tilde{\mathcal{W}}$ cannot have more than $\operatorname{dim}(\tilde{\mathcal{W}})$ linearly independent components. Based on the above results, we can now calculate this upper bound. It directly follows from the definition of $\mathcal{J}$ that $\operatorname{dim}(\tilde{\mathcal{W}})$ is given by the number of points $(n, k)$ such that both $n+k \in$ $\{0, \ldots, N-1\}$ and $n-k \in\{0, \ldots, N-1\}$. Combining $0 \leq n+k \leq N-1$ and $0 \leq n-k \leq N-1$ gives $-n \leq k \leq n$ 
if $0 \leq n \leq\lfloor(N-1) / 2\rfloor$, and $-(N-n-1) \leq k \leq(N-n-1)$ otherwise. Here, $\lfloor(N-1) / 2\rfloor$ denotes integer part of $(N-1) / 2$. Then, we have

$$
\begin{aligned}
\operatorname{dim}(\tilde{\mathcal{W}})=\sum_{n=0}^{\lfloor(N-1) / 2\rfloor}[2 n+1] & \\
& +\sum_{n=\lfloor(N-1) / 2\rfloor+1}^{N-1}[2(N-n-1)+1]
\end{aligned}
$$

from which we finally obtain that $\operatorname{dim}(\tilde{\mathcal{W}})$ equals $N^{2} / 2$ if $N$ is even and $\left(N^{2}+1\right) / 2$ if $N$ is odd. This result leads directly to the following theorem.

Theorem 1: Let $x$ and $y$ be $N$-sample complex-valued signals. The number of linearly independent samples $W_{x y}(n, m)$ of the discrete WD of $x$ and $y$ is upper bounded by $N^{2} / 2$ if $N$ is even and $\left(N^{2}+1\right) / 2$ if $N$ is odd.

Theorem 1 establishes that linear relations necessarily connect the samples $W_{x y}(n, m)$. This means that sets of generators $\left\{W_{x y}(n, m):(n, m) \in \mathcal{L}\right\}$ exist and are denoted $\mathcal{G}_{\mathcal{L}}$ so that every $W_{x y}(n, m)$ with $(n, m) \in\{0, \ldots, N-1\}^{2}$ can be expressed as a linear combination of the elements of $\mathcal{G}_{\mathcal{L}}$ for all $x, y \in \mathbb{C}^{N}$. Each set is completely determined by a family of TF locations $\mathcal{L}$ depending only on the linear signal space fixed beforehand, which is $\mathbb{C}^{N}$ here. To further derive the linear map $\mathbf{T}_{\mathcal{L}}$ connecting every $W_{x y}(n, m)$ with $\mathcal{G}_{\mathcal{L}}$, we now only need to characterize the elements of $\mathcal{G}_{\mathcal{L}}$.

We will now prove that $W_{x y}$ can be recovered from $\mathcal{G}_{\mathcal{L}}$ if, and only if, the latter contains $2 K^{(n)}+1$ linearly independent $W_{x y}(n, m)$ for each $n=0, \ldots, N-1$. Here, $K^{(n)}$ equals $n$ if $0 \leq n \leq\lfloor(N-1) / 2\rfloor$; otherwise, $K^{(n)}$ equals $N-n-1$. We observe that $R_{x y}(n, k)=0$ if $|k|>K^{(n)}$. Then, we have

$$
W_{x y}(n, m)=\sum_{k=-K^{(n)}}^{K^{(n)}} R_{x y}(n, k) \exp \left(-\frac{2 j \pi m k}{N}\right) .
$$

It can be verified that no linear relation connecting the cross correlation samples $R_{x y}(n, k)$ for all $x, y \in \mathbb{C}^{N}$ exists. With (4), this implies that the redundancy of information carried by the discrete cross WD consists of linear relations connecting the samples $W_{x y}(n, m)$ that have the same time-index $n$ for each $n=0, \ldots, N-1$. Throughout this paper, this redundancy will be called "spectral redundancy."
Let $n \in\{0, \ldots, N-1\}$ be a given time-index, and let $\mathbf{P}^{(n)}$ be the matrix whose $(m, k)$-component is given by

$$
\mathbf{P}^{(n)}(m, k) \triangleq \exp \left(\frac{2 j \pi m\left(k-K^{(n)}\right)}{N}\right)
$$

where $k \in\left\{0, \ldots, 2 K^{(n)}\right\}$, and $m \in\{0, \ldots, N-1\}$. Then, the discrete WD can be expressed as $W_{x y}^{(n)}=\mathbf{P}^{(n)} R_{x y}^{(n)}$ with

$$
\begin{gathered}
R_{x y}^{(n)} \triangleq\left[R_{x y}\left(n,-K^{(n)}\right) R_{x y}\left(n,-K^{(n)}+1\right) \cdots\right. \\
\left.R_{x y}\left(n, K^{(n)}-1\right) R_{x y}\left(n, K^{(n)}\right)\right]^{\mathrm{T}} \\
W_{x y}^{(n)} \triangleq\left[W_{x y}(n, 0) W_{x y}(n, 1) \cdots\right. \\
\left.W_{x y}(n, N-2) W_{x y}(n, N-1)\right]^{\mathrm{T}} .
\end{gathered}
$$

The superscript $\mathrm{T}$ denotes "transposition." Proceeding as in the proof of Theorem 1, it is a routine verification that the family $\left\{R_{\left.\Delta_{(n+k)} \Delta_{(n-k)}\right)}^{(n)}:|k| \leq K^{(n)}\right\}$ is a basis for the induced $R_{x y}^{(n)}$-domain space, which is denoted $\tilde{\mathcal{R}}^{(n)}$, of all linear combinations over the field $\mathbb{C}$ of local correlations $R_{x y}^{(n)}$, with $x, y \in \mathbb{C}^{N}$. From this, we conclude at once that the dimension of this space is $2 K^{(n)}+1$. Observe that $\operatorname{dim}(\tilde{\mathcal{W}})=\sum_{n=0}^{N-1}\left[2 K^{(n)}+1\right]$. Since $W_{x y}^{(n)}$ is the Fourier transform of $R_{x y}^{(n)}$, this implies that the dimension of the induced $W_{x y}^{(n)}$-domain space $\tilde{\mathcal{W}}^{(n)}$ is also $2 K^{(n)}+1$. Hence, $W_{x y}^{(n)}$ can be fully recovered from $\mathcal{G}_{\mathcal{L}}$ if the latter contains $2 K^{(n)}+1$ linearly independent components of $W_{x y}^{(n)}$.

Let $\left\{W_{x y}\left(n, m_{0}\right), \ldots, W_{x y}\left(n, m_{2 K^{(n)}}\right)\right\}$ be any set of $2 K^{(n)}+1$ distinct samples of $W_{x y}^{(n)}$. We will now prove that the elements of this set, which henceforth are denoted by $\mathcal{G}_{\mathcal{L}}^{(n)}$, are inevitably linearly independent. The vector $W_{x y}^{(n)}$ can be partitioned between $W_{x y, \mathcal{L}}^{(n)}$ and $W_{x y, \overline{\mathcal{L}}}^{(n)}$ in such a way that the components of $W_{x y, \mathcal{L}}^{(n)}$ are the elements of $\mathcal{G}_{\mathcal{L}}^{(n)}$. Then, the relation $W_{x y}^{(n)}=\mathbf{P}^{(n)} R_{x y}^{(n)}$ can be rewritten in the following form:

$$
\left(\begin{array}{c}
W_{x y, \mathcal{L}}^{(n)} \\
W_{x y, \overline{\mathcal{L}}}^{(n)}
\end{array}\right)=\left(\begin{array}{c}
\mathbf{P}_{\mathcal{L}}^{(n)} \\
\mathbf{P}_{\overline{\mathcal{L}}}^{(n)}
\end{array}\right) R_{x y}^{(n)}
$$

by reordering the rows of $W_{x y}^{(n)}, R_{x y}^{(n)}$, and $\mathbf{P}^{(n)}$, if necessary. Here, $\mathbf{P}_{\mathcal{L}}^{(n)}$ is the submatrix of $\mathbf{P}^{(n)}$ that verifies $W_{x y, \mathcal{L}}^{(n)}=$ $\mathbf{P}_{\mathcal{L}}^{(n)} R_{x y}^{(n)}$, namely, as shown in the equation at the bottom of the page. Since the $2 K^{(n)}+1$ components of $R_{x y}^{(n)}$ are linearly

$$
\mathbf{P}_{\mathcal{L}}^{(n)}=\left(\begin{array}{cccc}
\exp \left(\frac{2 j \pi m_{0}\left(-K^{(n)}\right)}{N}\right) & \exp \left(\frac{2 j \pi m_{0}\left(-K^{(n)}+1\right)}{N}\right) & \cdots & \exp \left(\frac{2 j \pi m_{0} K^{(n)}}{N}\right) \\
\exp \left(\frac{2 j \pi m_{1}\left(-K^{(n)}\right)}{N}\right) & \exp \left(\frac{2 j \pi m_{1}\left(-K^{(n)}+1\right)}{N}\right) & \cdots & \exp \left(\frac{2 j \pi m_{1} K^{(n)}}{N}\right) \\
\vdots & \vdots & & \vdots \\
\exp \left(\frac{2 j \pi m_{2 K^{(n)}}\left(-K^{(n)}\right)}{N}\right) & \exp \left(\frac{2 j \pi m_{2 K^{(n)}}\left(-K^{(n)}+1\right)}{N}\right) & \cdots & \exp \left(\frac{2 j \pi m_{2 K^{(n)}} K^{(n)}}{N}\right)
\end{array}\right)
$$


independent, it follows that the $2 K^{(n)}+1$ components of $W_{x y, \mathcal{L}}^{(n)}$ are also linearly independent if, and only if, $\mathbf{P}_{\mathcal{L}}^{(n)}$ is nonsingular. We will now evaluate the determinant of $\mathbf{P}_{\mathcal{L}}^{(n)}$. We may write it in the following form:

$$
\left|\mathbf{P}_{\mathcal{L}}^{(n)}\right|=V\left(m_{0}, \ldots, m_{2 K^{(n)}}\right) \prod_{i=0}^{2 K^{(n)}} \exp \left(-\frac{2 j \pi m_{i} K^{(n)}}{N}\right)
$$

with

$$
\begin{aligned}
& V\left(m_{0}, \ldots, m_{2 K^{(n)}}\right) \\
& =\left|\begin{array}{cccc}
1 & \exp \left(\frac{2 j \pi m_{0}}{N}\right) & \cdots & \exp \left(\frac{2 j \pi m_{0} 2 K^{(n)}}{N}\right) \\
\vdots & \vdots & \vdots \\
1 & \exp \left(\frac{2 j \pi m_{2 K^{(n)}}}{N}\right) & \cdots & \exp \left(\frac{2 j \pi m_{2 K^{(n)}} 2 K^{(n)}}{N}\right)
\end{array}\right| .
\end{aligned}
$$

We observe that $V\left(m_{0}, \ldots, m_{2 K^{(n)}}\right)$ is a Vandermonde determinant [12] whose value can be determined explicitly to be

$$
\begin{aligned}
& V\left(m_{0}, \ldots, m_{2 K^{(n)}}\right) \\
& =\prod_{0 \leq i<k \leq 2 K^{(n)}}\left[\exp \left(\frac{2 j \pi m_{k}}{N}\right)-\exp \left(\frac{2 j \pi m_{i}}{N}\right)\right] .
\end{aligned}
$$

Since $m_{i} \neq m_{k}$ for $i \neq k$, it follows that $V\left(m_{0}, \ldots\right.$, $\left.m_{2 K^{(n)}}\right) \neq 0$ and $\left|\mathbf{P}_{\mathcal{L}}^{(n)}\right| \neq 0$. Then, the components of $W_{x y, \mathcal{L}}^{(n)}$ are linearly independent. This implies that $W_{x y}^{(n)}$ can be recovered by choosing any $2 K^{(n)}+1$ distinct components $W_{x y}^{(n)}(m)$ for each $n=0, \ldots, N-1$. This property leads directly to the following theorem, which states a necessary and sufficient condition for the elements of any set of generators.

Theorem 2: Let $\left\{W_{x y}(n, m):(n, m) \in \mathcal{L}\right\}$ be a family of samples of $W_{x y}$, which is denoted $\mathcal{G}_{\mathcal{L}}$, where $x$ and $y$ are complex-valued signals of length $N$. $W_{x y}$ can be fully recovered from $\mathcal{G}_{\mathcal{L}}$, provided that $\mathcal{G}_{\mathcal{L}}$ contains at least $2 K^{(n)}+1$ distinct components $W_{x y}(n, m)$ for each $n=0, \ldots, N-1$. Here, $K^{(n)}$ equals $n$ if $0 \leq n \leq\lfloor(N-1) / 2\rfloor$; otherwise, $K^{(n)}$ equals $N-n-1$.

For every signal $x, y \in \mathbb{C}^{N}$, we can now characterize the linear relation $\mathbf{T}_{\mathcal{L}}$ between the samples $W_{x y}(n, m)$ of $W_{x y}$ and any set $\mathcal{G}_{\mathcal{L}}$ of generators satisfying the conditions of Theorem 2. Spectral redundancy enables us to restrict our attention to the linear map $\mathbf{T}_{\mathcal{L}}^{(n)}$ connecting the components of $W_{x y}^{(n)}$ to any set $\mathcal{G}_{\mathcal{L}}^{(n)}$ of $2 K^{(n)}+1$ distinct components $W_{x y}^{(n)}(m)$. By (6), we have

$$
W_{x y, \overline{\mathcal{L}}}^{(n)}=\mathbf{P}_{\overline{\mathcal{L}}}^{(n)} R_{x y}^{(n)}=\mathbf{P}_{\overline{\mathcal{L}}}^{(n)}\left(\mathbf{P}_{\mathcal{L}}^{(n)}\right)^{-1} W_{x y, \mathcal{L}}^{(n)}
$$

since the matrix $\mathbf{P}_{\mathcal{L}}^{(n)}$ is nonsingular. The expressions of $\mathbf{T}_{\mathcal{L}}^{(n)}$ and $\mathbf{T}_{\mathcal{L}}$ directly follow from the above equation.

\section{Example}

For the better understanding of the results established in Section II-B, we will now give an example where a set $\mathcal{G}_{\mathcal{L}}$ of generators is explicitly defined. In Fig. 2, we suppose that $N=8$, and we consider the TF samples represented by large dots. They are listed as follows.

$$
\begin{aligned}
n=0: & \mathcal{G}_{\mathcal{L}}^{(0)}=\left\{W_{x y}(0,3)\right\} \\
n=1: & \mathcal{G}_{\mathcal{L}}^{(1)}=\left\{W_{x y}(1,1), W_{x y}(1,2), W_{x y}(1,4)\right\} \\
n=2: & \mathcal{G}_{\mathcal{L}}^{(2)}=\left\{W_{x y}(2,0), W_{x y}(2,1), W_{x y}(2,2)\right. \\
& \left.W_{x y}(2,4), W_{x y}(2,5)\right\} \\
\cdots & \\
n=5: & \mathcal{G}_{\mathcal{L}}^{(5)}=\left\{W_{x y}(5,2), W_{x y}(5,3), W_{x y}(5,4)\right. \\
& \left.W_{x y}(5,5), W_{x y}(5,6)\right\} \\
n=6: & \mathcal{G}_{\mathcal{L}}^{(6)}=\left\{W_{x y}(6,2), W_{x y}(6,3), W_{x y}(6,4)\right\} \\
n=7: & \mathcal{G}_{\mathcal{L}}^{(7)}=\left\{W_{x y}(7,4)\right\} .
\end{aligned}
$$

Since $\operatorname{card}\left(\mathcal{G}_{\mathcal{L}}^{(n)}\right)=2 K^{(n)}+1$ for each $n \in\{0, \ldots, 7\}$, it follows from Theorem 2 that $W_{x y}$ can be fully recovered from $\mathcal{G}_{\mathcal{L}}=\bigcup_{n=0}^{7} \mathcal{G}_{\mathcal{L}}^{(n)}$. From the theory developed in the preceding subsection, we can get an explicit expression of the linear map connecting the components of $W_{x y}^{(n)}$, namely, $\left[W_{x y}(n, 0) \cdots W_{x y}(n, 7)\right]^{\mathrm{T}}$, to the elements of $\mathcal{G}_{\mathcal{L}}^{(n)}$ for each $n=0, \ldots, 7$. For example, using (7) with $n=1$ gives

$$
\begin{aligned}
& W_{x y, \overline{\mathcal{L}}}^{(1)} \\
& \overbrace{\left(\begin{array}{l}
W_{x y}(1,0) \\
W_{x y}(1,3) \\
W_{x y}(1,5) \\
W_{x y}(1,6) \\
W_{x y}(1,7)
\end{array}\right)} \\
& \overbrace{\left(\begin{array}{lll}
e^{(2 j \pi \cdot 0 \cdot(-1)) / 8} & e^{(2 j \pi \cdot 0 \cdot 0) / 8} & e^{(2 j \pi \cdot 0 \cdot 1) / 8} \\
e^{(2 j \pi \cdot 3 \cdot(-1)) / 8} & e^{(2 j \pi \cdot 3 \cdot 0) / 8} & e^{(2 j \pi \cdot 3 \cdot 1) / 8} \\
e^{(2 j \pi \cdot 5 \cdot(-1)) / 8} & e^{(2 j \pi \cdot 5 \cdot 0) / 8} & e^{(2 j \pi \cdot 5 \cdot 1) / 8} \\
e^{(2 j \pi \cdot 6 \cdot(-1)) / 8} & e^{(2 j \pi \cdot 6 \cdot 0) / 8} & e^{(2 j \pi \cdot 6 \cdot 1) / 8} \\
e^{(2 j \pi \cdot 7 \cdot(-1)) / 8} & e^{(2 j \pi \cdot 7 \cdot 0) / 8} & e^{(2 j \pi \cdot 7 \cdot 1) / 8}
\end{array}\right)} \\
& \underbrace{\left(\begin{array}{lll}
e^{(2 j \pi \cdot 1 \cdot(-1)) / 8} & e^{(2 j \pi \cdot 1 \cdot 0) / 8} & e^{(2 j \pi \cdot 1 \cdot 1) / 8} \\
e^{(2 j \pi \cdot 2 \cdot(-1)) / 8} & e^{(2 j \pi \cdot 2 \cdot 0) / 8} & e^{(2 j \pi \cdot 2 \cdot 1) / 8} \\
e^{(2 j \pi \cdot 4 \cdot(-1)) / 8} & e^{(2 j \pi \cdot 4 \cdot 0) / 8} & e^{(2 j \pi \cdot 4 \cdot 1) / 8}
\end{array}\right)^{-1}} \\
& \left(\mathbf{P}_{\mathcal{L}}^{(1)}\right)^{-1} \\
& \underbrace{\left(\begin{array}{l}
W_{x y}(1,1) \\
W_{x y}(1,2) \\
W_{x y}(1,4)
\end{array}\right)}_{W_{x y, \mathcal{L}}^{(1)}} .
\end{aligned}
$$

To get (8), the vector $W_{x y}^{(1)}$ has been partitioned between $W_{x y, \mathcal{L}}^{(1)}$ and $W_{x y, \overline{\mathcal{L}}}^{(1)}$ in such a way that the components of $W_{x y, \mathcal{L}}^{(1)}$ are the elements of $\mathcal{G}_{\mathcal{L}}^{(1)}$. The rows of $\mathbf{P}_{\mathcal{L}}^{(1)}$ and $\mathbf{P}_{\overline{\mathcal{L}}}^{(1)}$ have been extracted from the matrix $\mathbf{P}^{(1)}$ defined in (5) and reordered so that (6) is 


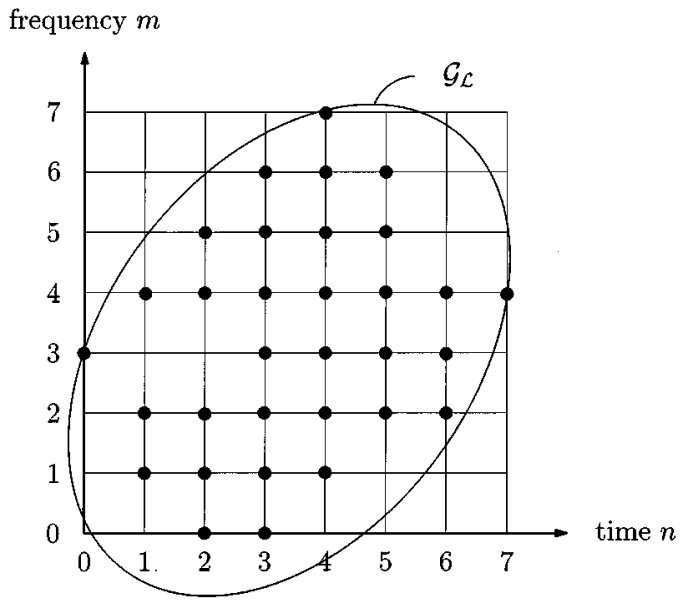

Fig. 2. Generators of $W_{x y}$ depicted schematically by large dots, for all $x, y \in$ $\mathbb{C}^{N}$.

verified by the vectors $W_{x y}^{(1)}$ and $W_{x y, \overline{\mathcal{L}}}^{(1)}$ fixed beforehand, and $R_{x y}^{(1)}=\left[R_{x y}(1,-1) R_{x y}(1,0) R_{x y}(1,1)\right]^{\mathrm{T}}$.

\section{LINEAR REDUNDANCY OF THE DISCRETE AUTO WD}

In the previous section, we have studied the redundancy of information encoded by the discrete cross WD in the case of complex-valued signals. This algebraic study can be applied to various situations. Two examples are proposed below.

\section{A. Discrete Auto-WD of Complex-Valued Signals}

The first example is concerned with the linear redundancy of information carried by the discrete auto WD of any complexvalued signal $x$. This is a special case of Section II-B, and we can check that the proofs of Theorems 1 and 2 are applicable to $W_{x}$. These results remain unchanged, and (7) still holds.

\section{B. Discrete Auto-WD of Real-Valued Signals}

The second example presented is concerned with the discrete auto-WD in the case where the signal of interest $x$ is an element of the linear space of real-valued signals of length $N$ over the field $\mathbb{R}$. To establish an upper bound to the number of linearly independent components of $W_{x}$, we proceed as we did in Section II-B. We first expand $W_{x}$ in the following form:

$$
W_{x}=\sum_{(n, k) \in \mathcal{J}} R_{x}(n, k) W_{\Delta_{(n+k)} \Delta_{(n-k)}} \cdot
$$

Since the signal $x$ is real-valued, it directly follows that $R_{x}(n, k)=R_{x}(n,-k)$ and

$$
\begin{aligned}
W_{x}= & \sum_{k=0}^{N-1} R_{x}(n, 0) W_{\Delta_{n}}+\sum_{\substack{(n, k) \in \mathcal{J} \\
k>0}} R_{x}(n, k) \\
& \cdot\left[W_{\Delta_{(n+k)} \Delta_{(n-k)}}+W_{\Delta_{(n-k)} \Delta_{(n+k)}}\right] .
\end{aligned}
$$

From this, we see that the elements of

$$
\left\{W_{\Delta_{(n+k)} \Delta_{(n-k)}}+W_{\Delta_{(n-k)} \Delta_{(n+k)}}:(n, k) \in \mathcal{J}, k \geq 0\right\}
$$

generate $W_{x}$ for all $x \in \mathbb{R}$. Furthermore, these generators are linearly independent since the elements of $\left\{W_{\Delta_{(n+k)} \Delta_{(n-k)}}:(n, k) \in \mathcal{J}\right\}$ are orthonormal. Then, it follows from the definition of $\mathcal{J}$ that the number of linearly independent components of $W_{x}$ is upper bounded by the number of points $(n, k)$ so that both $n+k \in\{0, \ldots, N-1\}$ and $n-k \in\{0, \ldots, N-1\}$ with $k \geq 0$. Combining these conditions yields $0 \leq k \leq n$ if $0 \leq n \leq\lfloor(N-1) / 2\rfloor$; otherwise, $0 \leq k \leq(N-n-1)$. Hence, $W_{x}$ cannot have more than

$$
\sum_{n=0}^{\lfloor(N-1) / 2\rfloor}[n+1]+\sum_{n=\lfloor(N-1) / 2\rfloor+1}^{N-1}[(N-n-1)+1]
$$

linearly independent components. This expression leads directly to the following theorem.

Theorem 3: For all $x \in \mathbb{R}^{N}$, the number of linearly independent components $W_{x}(n, m)$ of the discrete WD of $x$ is upper bounded by $\left(N^{2}+2 N\right) / 4$ if $N$ is even and $(N+1)^{2} / 4$ if $N$ is odd.

This result establishes that the discrete auto-WD encodes information carried by real-valued signals in a redundant fashion. In the light of Section III-A, we note the decrease in the number of linearly independent components $W_{x}(n, m)$ due to the symmetry property $R_{x}(n, k)=R_{x}(n,-k)$. Again, we can find sets of generators $\mathcal{G}_{\mathcal{L}}$ so that every component of $W_{x}$ can be expressed as a linear combination of the elements of $\mathcal{G}_{\mathcal{L}}$ for all $x \in \mathbb{R}^{N}$. The next theorem specifies how to construct such sets.

Theorem 4: Let $\left\{W_{x}(n, m):(n, m) \in \mathcal{L}\right\}$ be a family of components of $W_{x}$, which is denoted $\mathcal{G}_{\mathcal{L}}$, where $x$ is any realvalued signal of length $N$. $W_{x}$ can be fully recovered from $\mathcal{G}_{\mathcal{L}}$, provided that $\mathcal{G}_{\mathcal{L}}$ contains at least $K^{(n)}+1$ distinct samples $W_{x}(n, m)$ for each $n=0, \ldots, N-1$. As in Theorem $2, K^{(n)}$ equals $n$ if $0 \leq n \leq\lfloor(N-1) / 2\rfloor$; otherwise, $K^{(n)}$ equals $N-n-1$.

Theorem 4 is equivalent to Theorem 2 applied to the case $x=y$ with $x, y \in \mathbb{R}^{N}$. In the Appendix, the proof proposed in Section II-B is then used with a minor modification of the definition (5) to prove the above theorem. We conclude the Appendix with the expression of the linear map connecting the components of $W_{x}$ to any set of generators, which is of the form (7) applied to modified definitions for $\mathbf{P}_{\mathcal{L}}^{(n)}$ and $\mathbf{P}_{\overline{\mathcal{L}}}^{(n)}$.

\section{SimUlation EXAMPLES}

Let us now concentrate on computer simulations to illustrate the main theoretical results presented in previous sections.

\section{A. Experiment 1: Locations of Generators, Reconstruction}

Figs. 3 and 4 illustrate the redundancy of information carried by the discrete auto WD of complex-valued signals and real-valued signals, respectively. Theorems 2 and 4 were used to find locations of generators in the TF domain, which are represented in these figures by white colored areas. This means that information carried by black colored areas can be derived from information carried by white colored ones using linear transformations. The matrices associated with such linear transformations were computed as shown previously. Then, they were used 
(a)

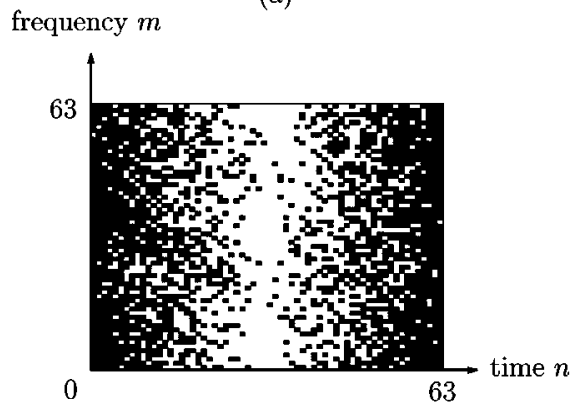

(b)

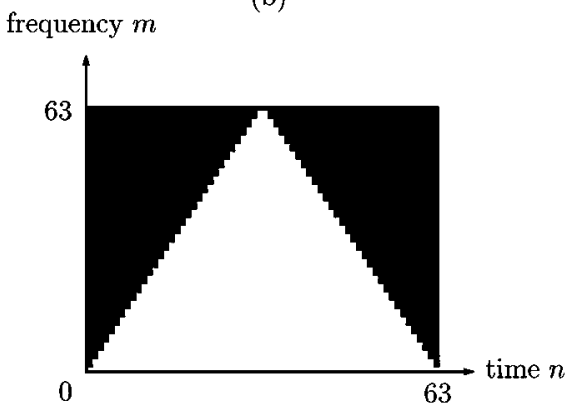

Fig. 3. Linear redundancy of information carried by discrete auto WD of 64-sample complex-valued signals. In (a) and (b), information carried by the black colored areas can be derived from information carried by the white colored ones using linear transformations.

(a)

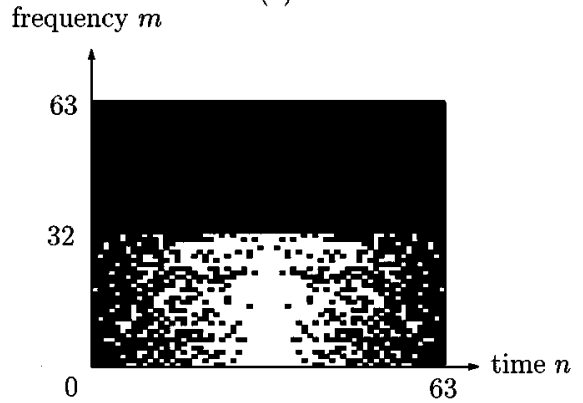

(b)

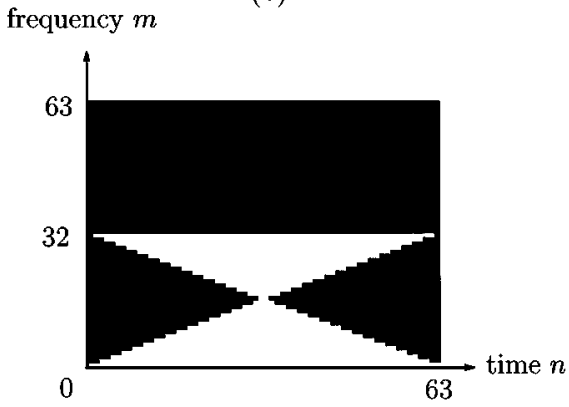

Fig. 4. Linear redundancy of information carried by discrete auto WD of 64-sample real-valued signals. In (a) and (b), information carried by the black colored areas can be derived from information carried by the white colored ones using linear transformations.

(a)

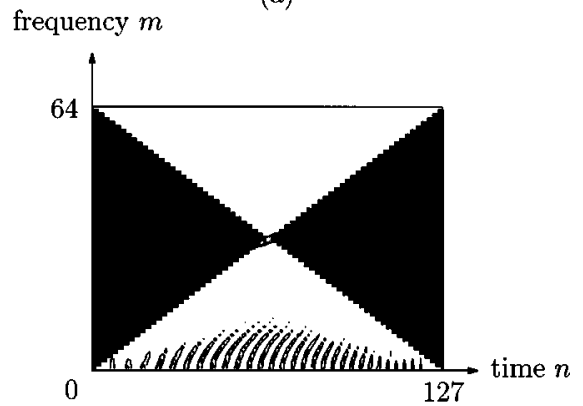

(b)

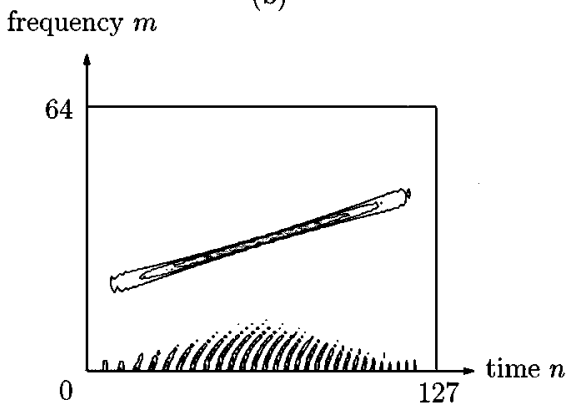

Fig. 5. Information carried by the set of generators depicted schematically in (a) by a white colored area is used to regenerate the discrete WD of a 128-sample real-valued chirp represented in (b).

to recover the discrete auto WD depicted in Fig. 5(b) from information carried by the generators represented in Fig. 5(a).

\section{B. Experiment 2: Detection in the TF Domain}

This example is concerned with the following detection scenario. Given a discrete-time real-valued signal $x$ received over the interval $\{0, \ldots, N-1\}$, we have to decide between the competing hypotheses $\mathrm{H}_{0}$ and $\mathrm{H}_{1}$ :

$$
\left\{\begin{array}{l}
\mathrm{H}_{0}: x=w \\
\mathrm{H}_{1}: x=s+w
\end{array}\right.
$$

where $s$ is the underlying signal to be detected and $w$ some additive noise. The decision between $\mathrm{H}_{0}$ and $\mathrm{H}_{1}$ is often made by comparing a test statistic $\lambda(x)$, computed from observation $x$, to some preset threshold $\lambda_{0}$. Here, we consider the general class of linear detectors $D$ operating in the whole TF domain

$$
D(x)=\left\{\begin{array}{cc}
1, & \text { if } \lambda(x)=\sum_{n=0}^{N-1} \sum_{m=0}^{N-1} W_{x}(n, m) V(n, m)>\lambda_{0} \\
\left(\text { decision } \mathrm{H}_{1}\right) \\
0, \quad \text { if } \lambda(x) \leq \lambda_{0}, \quad\left(\text { decision } \mathrm{H}_{0}\right)
\end{array}\right.
$$

where $V$ is a reference to be determined. Given $V$, we now wish to modify (12) so that the detector $D$ only exploits information carried by any set of generators $\mathcal{G}_{\mathcal{L}}$.

Let $\mathcal{G}_{\mathcal{L}}$ denote a set of generators. Let $W_{x}^{(n)}$ and $V^{(n)}$ correspond to the transposition of the $n$th row of the matrices $W_{x}$ and $V$, respectively. Then, the test statistic $\lambda(x)$ can be expressed as $\lambda(x)=\sum_{n=0}^{N-1} \lambda^{(n)}(x)$ with $\lambda^{(n)}(x)=\left\langle W_{x}^{(n)} \mid V^{(n)}\right\rangle$. Using the same notation for vectors $W_{x}^{(n)}$ and $V^{(n)}$ as Section II-B, 

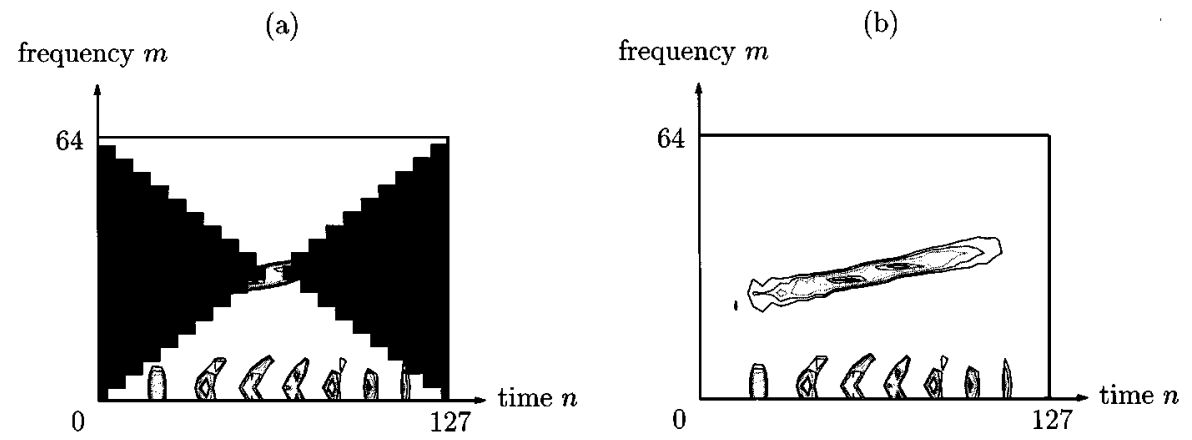

Fig. 6. Detector $D$ operating in the set of generators depicted schematically in (a) by a white colored area is designed to detect the presence or absence of a 128-sample real-valued chirp in white Gaussian noise (SNR $=0 \mathrm{~dB}$ ). The WD of this signal is given in (b).
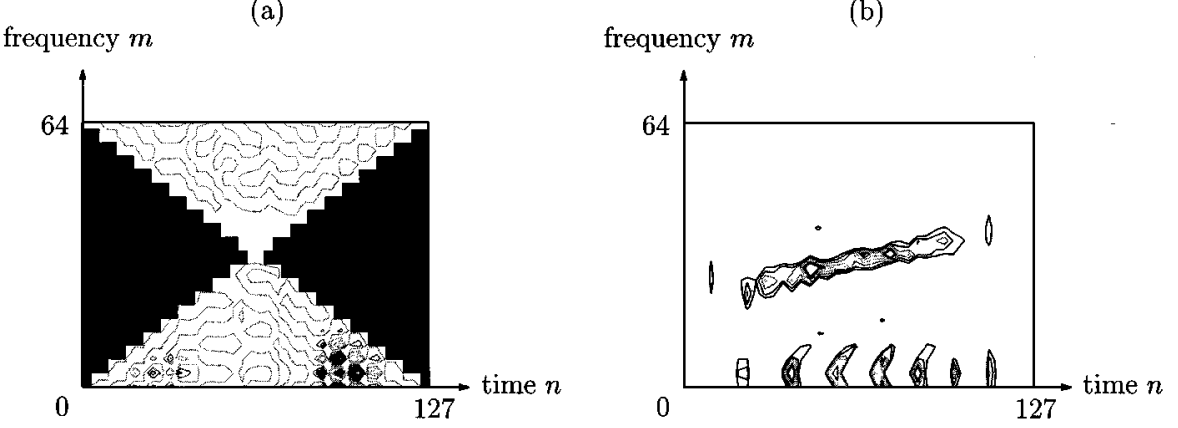

Fig. 7. Configurations of the detector $D$ operating in the set of generators represented in Fig. 6(a) or in the whole TF domain are given in (a) and (b), respectively.

$\lambda^{(n)}(x)$ can be decomposed as $\left\langle W_{x, \mathcal{L}}^{(n)} \mid V_{\mathcal{L}}^{(n)}\right\rangle+\left\langle W_{x, \overline{\mathcal{L}}}^{(n)} \mid V_{\overline{\mathcal{L}}}^{(n)}\right\rangle$ Assume that $W_{x, \overline{\mathcal{L}}}^{(n)}=\mathbf{T}_{\mathcal{L}}^{(n)} W_{x, \mathcal{L}}^{(n)}$. Then, we get

$$
\lambda^{(n)}(x)=\left\langle W_{x, \mathcal{L}}^{(n)} \mid V_{\mathcal{L}}^{(n)}+\left(\mathbf{T}_{\mathcal{L}}^{(n)}\right)^{\mathrm{T}} V_{\overline{\mathcal{L}}}^{(n)}\right\rangle .
$$

This equation indicates that every linear detector $D$ can be reformulated as

$$
D(x)=\left\{\begin{aligned}
& 1, \quad \text { if } \lambda(x)=\sum_{(n, m) \in \mathcal{L}} W_{x}(n, m) U_{\mathcal{L}}(n, m) \\
&>\lambda_{0} \\
& 0, \quad \text { if } \lambda(x) \leq \lambda_{0}
\end{aligned}\right.
$$

with $U_{\mathcal{L}}^{(n)} \triangleq V_{\mathcal{L}}^{(n)}+\left(\mathbf{T}_{\mathcal{L}}^{(n)}\right)^{\mathrm{T}} V_{\overline{\mathcal{L}}}^{(n)}$ for each $n=0, \ldots, N-1$. Conversely, every component of $V^{(n)}$ can be computed from $U_{\mathcal{L}}^{(n)}$ by solving the linear system

$$
U_{\mathcal{L}}^{(n)}=V_{\mathcal{L}}^{(n)}+\left(\mathbf{T}_{\mathcal{L}}^{(n)}\right)^{\mathrm{T}} V_{\overline{\mathcal{L}}}^{(n)}
$$

with $V_{\overline{\mathcal{L}}}^{(n)}=\mathbf{T}_{\mathcal{L}}^{(n)} V_{\mathcal{L}}^{(n)}$, for each $n=0, \ldots, N-1$, since

$$
\begin{aligned}
\left\langle W_{x, \mathcal{L}}^{(n)} \mid U_{\mathcal{L}}^{(n)}\right\rangle & =\left\langle W_{x, \mathcal{L}}^{(n)} \mid V_{\mathcal{L}}^{(n)}+\left(\mathbf{T}_{\mathcal{L}}^{(n)}\right)^{\mathrm{T}} \mathbf{T}_{\mathcal{L}}^{(n)} V_{\overline{\mathcal{L}}}^{(n)}\right\rangle \\
& =\left\langle W_{x, \mathcal{L}}^{(n)} \mid V_{\mathcal{L}}^{(n)}\right\rangle+\left\langle W_{x, \overline{\mathcal{L}}}^{(n)} \mid V_{\overline{\mathcal{L}}}^{(n)}\right\rangle \\
& =\left\langle W_{x}^{(n)} \mid V^{(n)}\right\rangle .
\end{aligned}
$$

An experiment of blind derivation of detectors solely from training data was conducted to illustrate configurations (12) and (14) of the detector $D$. In the case of detecting the presence or absence of the $N$-length signal $s(n) e^{j \phi}$ embedded in white Gaussian noise, with $s$ a deterministically known signal and $\phi \in[0,2 \pi]$ a uniform random variable, the optimal test statistic $\lambda(x)$ is known to be the inner product of the discrete WD of the observation $x$ with that of the signal to be detected [7], an example of which is shown in Fig. 6(b). First, detector (14), operating in the set of generators $\mathcal{G}_{\mathcal{L}}$ represented in Fig. 6(a), was designed as in [24] with 5600 realizations of the signal plus noise and noise only. The resulting reference $U_{\mathcal{L}}$ is shown in Fig. 7(a). Second, (15) was used to derive detector (12) from $U_{\mathcal{L}}$. The result represented in Fig. 7(b) conforms with our theoretical results since the reference $V$ closely resembles $W_{s}$. Obviously, configurations (12) and (14) yield the same performance.

\section{CONCLUSION}

An algebraic study of the linear redundancy of the information carried by the discrete cross WD has been proposed. We have shown that sets $\left\{W_{x}(n, m):(n, m) \in \mathcal{L}\right\}$ of generators exist (which are denoted $\mathcal{G}_{\mathcal{L}}$ ) so that every component $W_{x y}(n, m)$ with $(n, m) \in\{0, \ldots, N-1\}^{2}$ can be expressed as a linear combination of the elements of $\mathcal{G}_{\mathcal{L}}$ for all $N$-sample complex-valued signals $x, y$. We have formally identified the elements of $\mathcal{G}_{\mathcal{L}}$, and we have derived the linear map $\mathbf{T}_{\mathcal{L}}$ connecting every component of $W_{x y}$ with $\mathcal{G}_{\mathcal{L}}$. Finally, we have considered the special cases of discrete auto WD of complex-valued signals and real-valued signals, and we have illustrated the results by means of computer simulations. To complete the work begun in this paper, two important problems remain unsolved and are a potential ground for future research.

First, our results need to be extended to other linear signal spaces like that of analytic signals, which are currently used in TF analysis for improving the clearness and the readability of representations. Partial results have been obtained in [26] and 
[27]. For any analytic signal $x$ of even length $N$, it is shown that every component of $W_{x}$ can be expressed as a linear combination of $N^{2} / 4+N-1$ generators, but the problem, in its full generality, remains open since locations of generators in the TF domain have still not been identified.

Second, this study needs to be extended to other representations of Cohen's class. One direct application could be the control of the dimensionality of classifiers operating in the TF domain by selecting appropriate representations. Such results may be also a potential area of new research in applications like the suppression of interference terms, denoising, fast algorithms, and the resolution of signal synthesis problems.

\section{APPENDIX}

We will prove Theorem 4 by limiting the proof of Theorem 2 to the case $x=y$ with $x, y \in \mathbb{R}^{N}$. We first have to show that $W_{x}$ can be fully recovered from $\mathcal{G}_{\mathcal{L}}$ if, and only if, the latter contains $K^{(n)}+1$ linearly independent components $W_{x}(n, m)$ for each $n=0, \ldots, N-1$. Since $R_{x}(n, k)=0$ if $|k|>K^{(n)}$ and $R_{x}(n, k)=R_{x}(n,-k)$, we have

$$
W_{x}(n, m)=R_{x}(n, 0)+2 \sum_{k=1}^{K^{(n)}} R_{x}(n, k) \cos \left(\frac{2 \pi m k}{N}\right) .
$$

Let $n \in\{0, \ldots, N-1\}$ be a given time-index, and let $\mathbf{P}^{(n)}$ be the matrix whose $(m, k)$-component is given by

$$
\mathbf{P}^{(n)}(m, k) \triangleq \cos \left(\frac{2 \pi m k}{N}\right)
$$

with $m \in\{0, \ldots, N-1\}$ and $k \in\left\{0, \ldots, K^{(n)}\right\}$. Then, the discrete auto-WD of $x$ can be expressed as $W_{x}^{(n)}=\mathbf{P}^{(n)} R_{x}^{(n)}$ with

$$
\begin{gathered}
R_{x}^{(n)} \triangleq\left[R_{x}(n, 0) 2 R_{x}(n, 1) \cdots\right. \\
\left.2 R_{x}\left(n, K^{(n)}-1\right) 2 R_{x}\left(n, K^{(n)}\right)\right]^{\mathrm{T}} \\
W_{x}^{(n)} \triangleq\left[\begin{array}{l}
W_{x}(n, 0) W_{x}(n, 1) \cdots \\
\left.W_{x}(n, N-2) W_{x}(n, N-1)\right]^{\mathrm{T}} .
\end{array}\right.
\end{gathered}
$$

The proof given in Section II-B for the analog of Theorem 4 can be applied without modification to prove that the number of linearly independent components of $W_{x}^{(n)}$ is upper bounded by $K^{(n)}+1$.

Let $\left\{W_{x}\left(n, m_{0}\right), \ldots, W_{x}\left(n, m_{K^{(n)}}\right)\right\}$ be any set of $K^{(n)}+$ 1 distinct components of $W_{x}^{(n)}$. It remains to be proven that the elements of this set, which are denoted $\mathcal{G}_{\mathcal{L}}^{(n)}$, are linearly independent. The vector $W_{x}^{(n)}$ can be partitioned between $W_{x, \mathcal{L}}^{(n)}$ and $W_{x, \mathcal{L}}^{(n)}$ in such a way that the components of $W_{x, \mathcal{L}}^{(n)}$ are the elements of $\mathcal{G}_{\mathcal{L}}^{(n)}$. Then, the relation $W_{x}^{(n)}=\mathbf{P}^{(n)} R_{x}^{(n)}$ can be rewritten in the following form:

$$
\left(\begin{array}{l}
W_{x, \mathcal{L}}^{(n)} \\
W_{x, \overline{\mathcal{L}}}^{(n)}
\end{array}\right)=\left(\begin{array}{c}
\mathbf{P}_{\mathcal{L}}^{(n)} \\
\mathbf{P}_{\overline{\mathcal{L}}}^{(n)}
\end{array}\right) R_{x}^{(n)}
$$

by reordering the rows of $W_{x}^{(n)}, R_{x}^{(n)}$, and $\mathbf{P}^{(n)}$, if necessary. Here, $\mathbf{P}_{\mathcal{C}}^{(n)}$ is the submatrix of $\mathbf{P}^{(n)}$ such that $W_{x y, \mathcal{L}}^{(n)}=\mathbf{P}_{\mathcal{L}}^{(n)} R_{x y}^{(n)}$, namely

$$
\mathbf{P}_{\mathcal{L}}^{(n)}=\left(\begin{array}{cccc}
1 & \cos \left(\frac{2 \pi m_{0}}{N}\right) & \cdots & \cos \left(\frac{2 \pi m_{0} K^{(n)}}{N}\right) \\
1 & \cos \left(\frac{2 \pi m_{1}}{N}\right) & \cdots & \cos \left(\frac{2 \pi m_{1} K^{(n)}}{N}\right) \\
\vdots & \vdots & & \vdots \\
1 & \cos \left(\frac{2 \pi m_{K^{(n)}}}{N}\right) & \cdots & \cos \left(\frac{2 \pi m_{K^{(n)}} K^{(n)}}{N}\right)
\end{array}\right) .
$$

It can be verified in a manner similar to the one used in the proof of Theorem 2 that the elements of $\mathcal{G}_{\mathcal{L}}^{(n)}$ are linearly independent if, and only if, $\mathbf{P}_{\mathcal{L}}^{(n)}$ is a nonsingular matrix. To evaluate $\left|\mathbf{P}_{\mathcal{L}}^{(n)}\right|$ is a tedious calculus exercise leading to the result

$$
\begin{aligned}
\left|\mathbf{P}_{\mathcal{L}}^{(n)}\right|= & 2^{K^{(n)}\left(K^{(n)}-1\right) / 2} \prod_{0 \leq i<k \leq K^{(n)}} \\
& \cdot\left[\cos \left(\frac{2 \pi m_{k}}{N}\right)-\cos \left(\frac{2 \pi m_{i}}{N}\right)\right] .
\end{aligned}
$$

Then, $\left|\mathbf{P}_{\mathcal{L}}^{(n)}\right| \neq 0$ if, and only if

$$
\cos \left(2 \pi m_{k} / N\right) \neq \cos \left(2 \pi m_{i} / N\right) .
$$

This condition is ensured by $m_{i} \neq m_{k}$ and $m_{i} \neq N-m_{k}$ since $m_{i}, m_{k} \in\{0, \ldots, N-1\}$, i.e., $W_{x}\left(n, m_{i}\right) \neq W_{x}\left(n, m_{k}\right)$, thereby proving Theorem 4 . Finally, we note that the components of $W_{x, \mathcal{L}}^{(n)}$ can be deduced from $W_{x, \mathcal{L}}^{(n)}$ using (7) applied to the matrix $\mathbf{P}^{(n)}$ defined in this Appendix.

\section{ACKNOWLEDGMENT}

The author is grateful to Prof. R. Lengelle for his valuable comments and suggestions.

\section{REFERENCES}

[1] L. Atlas, J. Droppo, and J. McLaughlin, "Optimizing time-frequency distributions for automatic classification," Proc. SPIE, vol. 3162, 1997.

[2] T. Claasen and W. Mecklenbräuker, "The Wigner distribution-A tool for time-frequency signal analysis-Part II: Discrete-time signals," Philips J. Res., vol. 35, pp. 276-300, 1980.

[3] L. Cohen, Time-Frequency Analysis. Englewood Cliffs, NJ: PrenticeHall, 1995.

[4] A. Costa and G. Faye Boudreaux-Bartels, "An overview of aliasing errors in discrete-time formulations of time-frequency representations," IEEE Trans. Signal Processing, vol. 47, pp. 1463-1474, May 1999.

[5] J. Droppo and L. Atlas, "Application of classifier-optimal time-frequency distributions to speech analysis," in Proc. IEEE-SP Int. Symp. Time-Frequency Time-Scale Anal., Pittsburgh, PA, 1998, pp. 585-588.

[6] P. Flandrin, Time-Frequency/Time-Scale Analysis. San Diego, CA: Academic, 1999.

[7] - "A time-frequency formulation of optimum detection," IEEE Trans. Acoust., Speech, Signal Processing, vol. 36, pp. 1377-1384, May 1988.

[8] G. Golub and C. Van Loan, Matrix Computations. Baltimore, MD: John Hopkins Univ. Press, 1993.

[9] F. Hlawatsch, Time-Frequency Analysis and Synthesis of Linear Signal Space. Norwell, MA: Kluwer, 1998.

[10] F. Hlawatsch and W. Krattenthaler, "Bilinear signal synthesis," IEEE Trans. Acoust., Speech, Signal Processing, vol. 40, pp. 352-363, Feb. 1992. 
[11] R. Horn and C. Johnson, Topics in Matrix Analysis. New York: Cambridge Univ. Press, 1994.

[12] S. Lang, Algebra. Reading, MA: Addison-Wesley, 1993.

[13] N. Marinovich, "The singular value decomposition of the Wigner distribution and its applications," in The Wigner Distribution: Theory and Applications in Signal Processing, W. Mecklenbräuker and F. Hlawatsch, Eds. Amsterdam, The Netherlands: Elsevier, 1997, pp. 319-373.

[14] N. Marinovich, W. Smith, R. Waag, and K. Parker, "Limits imposed by tissue on SVD-Wigner filtering to obtain the intrinsic frequency variation of ultrasonic echo waveforms," in Proc. IEEE Ultrason. Symp., 1989, pp. 945-950.

[15] N. Marinovich and G. Eichmann, "An outer product expansion of Wigner distribution," in Proc. IEEE Int. Conf. Acoustics, Speech, Signal Process., Tampa, FL, 1985, pp. 1021-1024.

[16] — - "Feature extraction and pattern classification in space-spatial frequency domain," Proc. SPIE, vol. 579, pp. 19-26, 1985.

[17] N. Marinovich and W. Smith, "Suppression of noise to extract the intrinsic frequency variation from an ultrasonic echo," in Proc. IEEE Ultrason. Symp., San Francisco, CA, 1985, pp. 841-846.

[18] W. Mecklenbräuker and F. Hlawatsch, Eds., The Wigner Distribution: Theory and Applications in Signal Processing. Amsterdam, The Netherlands: Elsevier, 1997.

[19] A. H. Nutall, "Alias-free Wigner distribution functions and complex ambiguity functions for discrete-time samples," Naval Underwater Syst. Cent., Newport, RI, Tech. Rep. 8533, 1989.

[20] J. O'Neill, P. Flandrin, and W. Williams, "On the existence of discrete Wigner distributions," IEEE Signal Processing Lett., vol. 6, pp. 304-306, Oct. 1999.

[21] J. O'Neill and W. Williams, "Shift covariant time-frequency distributions of discrete signals," IEEE Trans. Signal Processing, vol. 47, pp. 133-146, Jan. 1999.

[22] F. Peyrin and P. Prost, "A unified definition for the discrete-time discrete-frequency, and discrete-time/frequency Wigner distributions," IEEE Trans. Acoust., Speech, Signal Processing, vol. ASSP-34, pp. $858-867,1986$.
[23] C. Richard and R. Lengellé, "On the linear relations connecting the components of the discrete Wigner distribution in the case of real-valued signals," in Proc. IEEE Int. Conf. Acoust., Speech, Signal Process., Istanbul, Turkey, 2000, pp. 85-88.

[24] _ "Data-driven design and complexity control of time-frequency detectors," Signal Process., vol. 77, pp. 37-48, 1999.

[25] — - "Two algorithms for designing optimal reduced-bias data-driven time-frequency detectors," in Proc. IEEE-SP Int. Symp. Time-Frequency Time-Scale Anal., Pittsburgh, PA, 1998, pp. 601-604.

[26] — " "On the dimension of the discrete Wigner-Ville transform range space," in Proc. IEEE-SP Int. Symp. Time-Frequency Time-Scale Anal., Pittsburgh, PA, 1998, pp. 5-8.

[27] C. Richard, "Une méthodologie pour la détection à structure imposée. Applications au plan temps-fréquence," Ph.D. dissertation, Univ. Technol. Compiègne, Compiègne, France, 1998.

[28] M. Richman, T. Parks, and R. Shenoy, "Discrete-time, discrete-frequency, time-frequency analysis," IEEE Trans. Signal Processing, vol. 46, pp. 1517-1527, June 1998

[29] V. Vapnik, Statistical Learning Theory. New York: Wiley, 1998.

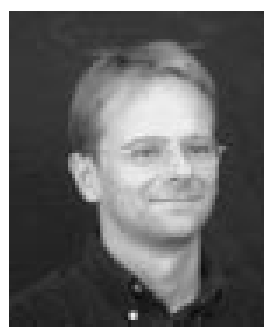

Cédric Richard (M'01) was born in Sarrebourg, France, on January 24, 1970. He received the Dipl.-Ing. and the M.S. degrees in 1994 and the Ph.D. degree in 1998 from Compiègne University of Technology, Compiègne, France, all in electrical and computer engineering.

Since 1995, he has been with the Systems Modeling and Dependability Laboratory, Troyes University of Technology, Troyes, France, where he is currently an Associate Professor with the Department of Information Systems Engineering. His current research interests involve time-frequency analysis, statistical estimation and decision theories, and pattern recognition. 